\title{
Beiträge zur Kunde des romanischen Elements im Serbokroatischen.
}

1. brgud Gemeindehutweide auf der Insel Veglia (kroat. $k r k$ ), s. Milčetić, Zbornik (Beiträge zur Kunde des Volkslebens und der Volkssitten) IX, 17, Mažuranić, Wbch. zu den Rechtsdenkmälern (kroat.) 100, Ak. Wb. I, 639, seit dem 15. Jh. auf derselben Insel und in Istrien belegt, so im Statut von Castua, Mon. hist.-jur. IV, 195, 62, wo man "lesi i brguda" "Wälder und Hutweiden" unterscheidet. Parčic, Vocab. croato-ital. übersetzt pascolo selvatico e rupestre. Aus dem Belege a. 1470 bei Surmin, Mon. croat. 259 ersieht man, dals so ein Teil von drmun, Wald' heifst. Auch als Ortsname, I. in Dalmatien bei Benkovac, a. 1396 Brigud geschrieben, heute Brgucl. 2. Die noch älteren Belege zeigen $-t$ für $-d$ : in Berguto a. I 186 bei Smičiklas, Cod. dipl. II, 205, heute Brgud bei Vrbnik (Veglia). 3. In Istrien Brgud a. 1546 Bergud. u geht auf älteres $u l$ zurück, wie der Beleg •a. I 188 a rivo de Zaulo (vegliotisch für Julius) usque Burguldum zeigt, Smičrklas o. c. II, 230; Jireček, Romanen I, 05. Davon dem. brgudac, Mon. croat. 245, auch als Ortsname auf der Insel Vis (Lissa) Brgujac. $j$ für $d$ ist skr! dialektische Erscheinung (aus dem Gen. -dca > -jca cf. Captajka, Biograjka). Es ist regelrechte Entsprechung des lat. virgultum.

2. bùrāg venter animalis $\mathrm{Ak}$. Wbch. I, 739, in östlichen Gegenden des skr. Sprachgebietes, deshalb bisher nur in Vuk's Wbch. gebucht, davon vb. proburáziti den Bauch durchstechen, BrozIveković o. c. II, 243. Es ist zu vergleichen mit burac derselben Bedeutung in der Handwerkersprache von Osatica (Bosnien), Glasnik (Mitteilungen des bos. Landesmuseums) XII, 59o. Als hypokoristische Bildung ist büro ventriosus aufzufassen, davon wiederum adj. bürav. Es ist nicht ausgeschlossen, dafs in diese Sippe auch rum. burtă, borf Bauch gehört, falls in $-t a,-f$ slaw. Suffixe stecken. Leider ist dies letztere im Slaw. selbst nicht zu belegen. Die Wörter gehören offenbar der Hirtensprache an. Die Grundlage ist mgl. buric Magen, buricos $=c u$ burtă (Delamatra) < umbilicus Puşc. 240. Der Grund für den Suffixwechsel $-i k>-\bar{a} g$ ist nicht klar.

3. celega, Art Decke für Barken, Waren; in den lateinisch geschriebenen Urkunden Ragusas, Jireček, Romanen I, 89. Für -ga steht -cha in der Schreibung zellicha. Es handelt sich also um 
denselben Lautübergang wie in astacus $>$ skr. jastog. Wie die Glosse celigas seu sclavinas (= härenes Gewand) zeigt, muls man das Wort mit prov. celetz < cilicium REW 1912 zusammenstellen. Die Endung wurde an serica angelehnt.1

4. Auch für das Skr. sind wie für das Ital. zwei Formen für Quitte anzunehmen, die eine mil $t$ und die andere mit $d^{2}: 1$. cotonea oder citonia (cytonea), 2. cydonea (cf. mala qudenea im dioklezianischen Maximalpreisetarif CIL III, S. 830, 6, $73=$ Blummer, Edictum Diocletiani de pretiis S. 98); vgl. dazu Plinius n. h. XV, 37 mala quae vocamus cotonea et Graeci cydonea. Danach wäre I. lat. und 2. griech., cf. Lindsay, Lat. Spr. 86. Auf 1. gehen zurück: mrkatuna im Ragusa ZfrPh. XXXVIII, 545, tkuńa mit der Umstellung $k t>t k$ wie in latak für lakat und $k l o>t k o$; mit dem Ausfall von $t$ in der Konsonantengruppe im Anlaut: küna ib. 5. 788, in Istrien küñva ib. 5, 789 ebenda oder mit dem von $k$ : tüńa in Sichelburg; auf 2. wiederum: gdüna ${ }^{3}$ ib. 3,126 seit dem 14. u. 15. Jh. belegt, mit dem Ausfall von $g$ : dinna ib. 2, 889 oder mit der Unstellung von $g d>d g$ wie in $d g e<g d \check{e}: d g u n a$ in Bačka und zuletzt mit dem Ausfall wie in $g d o>d o$ in Sichelburg. guńa ib. 3, 502, auch Ortsname in Slavonien; cüna in Rijeka (Fiume) und hüna in Istrien ib. I, 859. UI, 735 sprechen auch für 1., denn cuña beruht offenbar auf *cbtuha < citonea bei Anthimus (cf. nr. 14), welche Form über Umstellung und Ausfall, gerade so wie die oben angeführten, zu der heutigen gelangte, huńa wiederum auf ktuna $>{ }^{*}$ htuna $>{ }^{*}$ "thuna (vgl. für den Ausfall des $t$ - und z. T. parallele Entwicklung dzhorb $>$ tzhorb Iltis $>$ slov. thôr, skr. tvôr, for (Bosnien, Bańa Luka), vdrić (Kroatien, Bović), tōräc (Sichelburg) über *htorac). Ôber die geographische Verbreitung sind wir leider mangelhaft unterrichtet, nur soviel steht fest, dafs die $t$-Formen in dem dem Romanischen näheren Westen gebräuchlich sind. kuta, kutina, -ña, -ñaža, die auf dem kajkavischen (nördlichen) und slovenischen Gebiete vorkommen, sind keine romanischen, sondern ahd. Lehnwörter, $<$ ahd. kulina, bayr. Kütten. Cf. Berneker o. c. 299, wo die auf I beruhenden skr. Formen durch progessive Assimilation erklärt werden, wogegen aber die Geographie und cüha sprechen.

5. drkmar uncino, rampino (con quattro rebbj) bei Parčić o. c., auch drdkmār in Norddalmatien, tr̈kmār bei Spalato und Kaštela, auf der Insel Vrgada bei Zara, entspricht genau dem vegliot. dreknul REW 8836. Beide gehen auf *traginarius 4 zurück, nur ist im

1 Diese Ausgleichung der Endungen beider Wörter wurde vielleicht durch die $k$-Aussprache von ci begünstigt, vgl. comercium $>$ kumjerak in Ragusa, Ak. Wb. S. 779. In rellicha wäre dann ce wie in captat behandelt, vgl. unten Nr. 14.

$2 \mathrm{Vgl}$. aufser REW 2436 noch nuor. kitsnza, log. kidonsia, camp. tidonğa LbfrgPh. XXXIX, I3I.

8 für $c$ ist Assimilationsprodukt, wie in $k z d{ }^{k}>$ skr. $g$ dje, deshalb ist rum. gutuiü < cotōneu nur im Anfangskonsonant vom Slav. beeinflufst.

- Vgl. für die Erhaltung des $g$ vor $e, i$ wie hier noch von den Ortsnamen 1. Bag im kroat. Küstenland $<B i g i$ bei Ravennas, Ptol. Ovieyia, 
Vegliot. noch die bekannte Dissimilation eingetreten. Für skr. $\mathrm{km}$ $<k n$ vgl. poěmem für počnem; $d$ für $t$, sowie $k$ für $g$ sind unklar (Metathese der Stimme?).

6. fijersa, das Gesicht entstellende Narbe (Wundmal), in Ragusa, Ak. Wbch. III, 52 . Zu nordital. fersa $\mathrm{ZfrPh}$. XL, 109-12, venez. fersa sorta d'infermità che viene alla pelle (Boerio), friaul. fersa varuscli (Pirona).

7. gòstara lagoena vitrea Ak. Wbch. 3.323, dem. -ica, -icica, augm. -ina, gùsta(e)rica und mit unklarem $k$ (vielleicht nach kotarica Korb) kostarica ib. 5,373 $>{ }^{*}$ gastraria, prov. engrestara, aital. inguastara, it. guastada ( $d$ beruht auf der Dissimilation $r-r$, daher ist der Schwund des ersten $r$, entstanden etwa unter Einflufs von

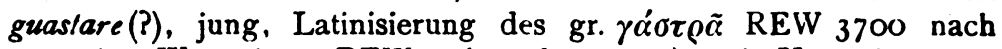
aquarium Wasserkrug REW 576,3 , bacar etc.); mit Umstellung vor $r$ auch im skr. grästa, -ica Ak. Wb. 3,389.

8. grànār Kornkammer bei den Ragusanern des 15 . und 16. Jh. Ak. Wbch. 3,385 , mit dem Suffixwechsel gìnao, gen. -ála ib. 3,453, entstanden wohl durch die Mittelstufen *garnar $>{ }^{*}$ grnar und durch die Dissimilation grnal; < granarium REW 3839 .

9. gùntura Beule hinter dem Ohr oder unter der Zahnlade, in der Lika (Kroatien) Ak. Wb. 3, 591, davon mit unklarem Suffix guntùrāc (kaum nach gluhać, da hier -ać zur Ableitung von nomen actoris dient) Rotzkrankheit der Pferde, gontinar todbringende Beule in der Bocche di Cattaro (Grbal) und Montenegro. $n$ ist Einschub-n vor Dentalen, cf. Strekelj, Zur slaw. Lehnwörterkunde 9, 78: < rum. gutunar sm., gutunări vb. (= guturaiü) Schnupfen $<*_{\text {gutturalium }}$ Puşc. 751, siz. gulturu Kropf REW 3930.

10. hrg situla, aqualis, dazu in Dalmatien h̆̈ga vaso di sasso per spulare orzo Parčić o. c. und Ak. Wbch. 3, 694. $\mathrm{Zu}$ orca REW 6087 und dieses zu v̋ $\varrho \chi \eta$ terrine (Boisacq, Dict. de la 1. grecque 1006). In diese Sippe können auch noch rum. hîrca Schädel und skr. kr̆ga Schöpfgefäls aus Kürbis, Berneker o. c. 666, gehören. Beim letzteren wäre noch Finflufs von $k r b, k r b u l a$ Körbchen (Berneker, o. c. 568) anzunehmen.

11. incéfo sm. gen. -ála antependium (neben pridotarnik) Altarvorhang, in Ragusa, Ak. Wb. 3,834, gehört zu rum. infáfa Kissen etc. überziehen und geht zurück auf *in-(oder ante)faciale. Das letztere findet Anschlufs an it. fazzoletto für $*$ fazzaletto, wo $o$ für $a$ auf derselben Angleichung beruht wie $i$ für $a$ in allrimenti. Für die Metathese $f-c>c-f$ vgl. das rum. Lehnwort nacafă für nafaca, oder skr. krlep für krpel, slov. makacal < ven. mazzocola, Strekelj, o. c. 37. Das Wort ist wohl ein Lehnwort aus dem Altdalmatischen, wie $a>e$, obwohl unbetont, vermuten lärst.

Plinius Vegium und meine Ausführnngen dazu im Glasnik des bos. Landesmuseums XXXI, 153; 2. Uglan Inselname bei Zara < im Mittelalter Gelanum, Nastavni Vjesnik XXIV, $659 \mathrm{f}$. 
I 2 intačiti perseverare, domandar con insistenza, con importunità (Parčić o. c.), adj. intǎ̌an lästig, gehört dem östlichen Gebiete, namentlich Slavonien und Serbien, an; entspricht dem rum. intefesc cu rugaciunt drängen, Puşc. 882.

13. jàbušoliti mit schwerer Mühe gehen, Ak. Wbch. 4, 390, dojabušoliti langsam und mit gröfster Mühe kommen, ib. 2, 580, in der Lika (Kroatien), also in einer Gegend, wo auch sonst in der Hirtensprache rum. Lehnwörter konstatiert wurden, s. AfslPh. 37, 8 If. Das Zeitwort beruht auf einer Umgestaltung des rum. Adv. (umbla) de-a bussele, a-busile auf allen Vieren gehen, Tiktin, Rum.-dtsch. Wbch. I, 492.

14. kîrla pennello da calafato, Ak. Wbch. 5,5 und Parčić o.c. Dieser Marinefachausdruck entsammt gewils dem Altdalm., wie schon die Erhaltung von $k$ vor $i$ zeigt, cf. $k \ddot{r} k l o$ Falte um die Kleidung, um dieselbe zu verkürzen, < circulus ib. 5, 59, Jaclan Inselname bei Ragusa, < Liciniana (insula), Makirina Flurname bei Spalato (cf. Bullettino di stor. e archeol. dalmata XXV, 189) < maceria + slav. Augmentativsuffix -ina, kapula < cepulla, kimak $<$ cimice, $k r f u l i c a$ ib. $5,530=$ slov. (Pleteršnik) krbulica $=$ krvel $=$ trbula (cf. trešńa für crešńa < ceresea) < caerefolium, lukijerna $<$ lucerna, rákno sorta di scialle (da donna) in der Umgebung von Spalato, Parčic o. c., Broz-Ivekovi\& o. c. II, $298<*_{\text {racenu für rachena }}$ (Georges), Krknata Inselname < circinata Nastarni Vjesnik XXIV, 66r. kirla geht auf *cirrula von cirrus, REW 1949, zurück. Wenn neben $k$ im Skr. noch $c$ (sprich $t s$ ) als Reflex des Lat. $c^{e i}$ erscheint, wie beispielsweise in Captat < Civitate, mocira < maceria, cresha dialektisch für trě̌na $<$ ceresea, ocat < acētum, ocèl <*aciale, 1 cer $<$ cerrus, cesar < Caesar, Cres (Cris) Inselname < Cherso, so spricht auch diese Entwicklung nicht gegen die $k$-Aussprache im Balkanlatein. Man wird vielmehr auch für das lat. $k$ zweifache Wiedergabe im Slaw. anzunehmen haben, genau so wie es für das ahd. $k$ vor $i$ notwendig ist, cf. skilling < aksl. skblezb, kroat. aber clez wie in corky < ahd. kirihha.

15. kò(u)nastra Biest, erste Milch von der Wöchnerin oder Haustieren nach dem Werfen, in der Landschaft Polica bei Spalato (private Mitteilung) $<$ colostra. $n$ statt $l$ erinnert an colucula $<$ frz. quenouille. Daneben kùlizdra, Schmähwort für ordinären Käse in der Lika (Kroatien), Ak. Wbch. 5, 770, wohl Entstellung des rum. colastrd.

16. kìzati onerare (navem), imponere (in navem) wird im Ak. Wbch. 5, 492 und so jetzt überall (cf. Mažuranić, o.c. 537) auf carricare zurückgeführt. Dabei bleibt aber $c$ (sprich $t s$ ) für

1 Vgl. von den Ortsnamen noch 1. slov. Ceje, deutsch Zilli < loc. Celeiae und dazu meine Ausführungen im Glasnik (Mitteilungen des bos. Landesmuseums) XXIX, 14I; 2. Srědectz (sprich Srjádetz), bei den Byzantinern Tralitza oder Triaditza < loc. Sardicae (sc. civitatis), von der trakischen Völkerschaft Sardi, heute Sofia (Bulg.). 
lat. ca ganz unerklärt, cf. Miklosich, Et. Wbch. 155 s. v. kürza. Die ricthtige Grundlage dieses Marinefachausdruckes kann nur carridiare sein; carrizare kommt in dalmatinischen Rechtsdenkmälern in derselben Bedeutung massenhaft vor, carizata Fahrstralse, Jireček, Romanen 1, 89. Die Wiedergabe des $d i$ durch $c$ wie im ital. olezzo, mezzo, rozzo.

17. kr̀puša Schafzecke, in Bosnien, in der Bocche di Cattaro und sonderbarerweise auch in Bakar (kroat. Küstenland), Ak. Wbch. $5,6 j \mathrm{O}$, ist ein rum. Lehnwort: căpuşă ds., davon încăpusa vb. knospen, wie das Vb. zeigt, Dem. von cap $+-u s ̧ a ̆$ (s. Puşc., Deminutivsuffixe $\S$ 166). Die skr. Wiedergabe des rum. $\breve{a}$ durch $r$ erinnert lebhaft an dieselbe des türk. $y(\varepsilon)$, z. B. skr. sìklet < türk. seklet, cf. AfslPh. $\mathrm{XXXV}, 346$.

18. lanterna REW 4896 hat im Skr. verschiedene Entsprechungen, die sich durch verschiedenes Alter der Entlehnungen erklären. 1. lùtērna bei einigen dalmatinischen Schriftstellern, Ak. Wbch. 6,191 mit $a{ }^{\text {cons. }}>u$ wie in sanctus $>$ sut. $c$ geht auf $t j$ zurück, da $e>j e$ wie in tovijerna $>$ taberna behandelt wurde. Das Wort hat somit mit lucerna nichts zu tun, wie Budmani a.a. O. will. Daneben lućerńa, da -na in Fremdwörtern oft zu tha wird, cf. štêrna < cisterna in Sichelburg. In Sichelburg lutêrña bei den Katholiken, lutig̨rna bei den Unierten. Als jüngeres Lehnwort ist dagegen ldtirna Ak. Wbch. 5, 897 (neben lanterna) zu betrachten; auch mit dem Schwund des als Artikel gefühlten l- und der Dissimilation $n-n>l-n$ : altirna bei einem Schriftsteller des 16. Jh., ib. I, 77, ferner mit dem Ersatz des an- durch das gewöhnlichere in-: intijerna, inle(i)rna in Ragusa, ib. 3,850.

19. mrča Myrte in Ragusa und anderswo in Dalmatien. \& für $t$ erklärt sich am besten durch -eus-Ableitung: *myrlea. Auch myrta REW 5801 kommt in Vraniic bei Spalato in der Gestalt mrtva vor, Ak. Wbch. 7,88. Dem altital. mortina REW 5803 entspricht mrtina, ib. 7,88 . Prov. nerlo erklärt sich durch die Angleichung des labialen Nasals an den Dental der folgenden Silbe, eine Erscheinung, die sowohl auf dem slaw. als auch auf dem roman. Gebiete konstatiert werden kann: skr. nicina neben micina Geschwür < rum. miałă Fieber <*ignztia von ignis (über diese Etymologie s. Casopis pro mod. fil. a lit. VII); skr. mito lautet in Sichelburg nito; aksl. mrèstz > skr. nërist, nerast; ${ }^{*}$ mitius in ital. Dialekten $>$ nizzo REW 5614, malta > nalta REW 5424 .

20. mugàra Schaf, welches nicht trächtig wurde, also ohne Lamm geblieben ist und somit weiter gemolken werden kann, in Istrien Ak. Wbch. 7, I 3 I. Es entspricht regelrecht einem lat. Adj. *mulgaris, wovon mulgare Melkgefäls überliefert ist. Eine andere Entsprechung von demselben Adj. ist gewils auch mrgar = margar Zbornik (Beiträge zur Kunde des Volkslebens und Sitten) V, 250, in den Rechtsdenkmälern seit dem 15. Jh. in Istrien und auf den Quarneroinseln mehrfach belegt. Es bedeutet eine Abteilung in 
dem mosuna < mansione benannten Schafstall, wo gemolken wird. Bezüglich des Übergangs von $u l$ cons. $>u r>$ skr. $r$ ist an rum. mursă < mulsa REW 5733 zu erinnern.

21. munčjela oder mučla Ak. Wbch. 7, 152, ein nicht näher bezeichnetes Wassesgefäls, ist eine weitere skr. Entsprechung von modiolus $\mathrm{ZfrPh}$. XXXVI, 6512, 16. Es entspricht dem vegliot. minzuol. Der Suffixwechsel -ellus statt -olus ist infolge der skr. Lauterscheinung $0>$ e nach Palatalen erfolgt. REW 4072.

22. ostil asta della fiòcina, astile (Parčić o. c.), zu hasta

23. párlog verwahrloster Weinberg, in Serbien, Broz-Iveković o. c. II, 12. Auch als Ortsname: Mali Parlozi in Serbien (Kreis Belgrad), Ak. Wbch. 6, 418. Dieses Wort beweist, dafs es auch im Skr. slaw. Rückentlehnungen aus dem Rum. gibt. Es ist rum. pirloch Brache < slav. prelogi, Tiktin, Deutsch.-rum. Wbch. I1 7 I.

24. plâg, Appellativ und Flurname zugleich auf der Insel Arbe (private Mitteilung), heilsen kleine Ebenen unter den Bergen. Die sich da sefshaft gemachten Einwohner werden Plajari genannt. Es entspricht dem rum. plaiu REW 6564.

25. plogar versante, auf der Insel Arbe (private Mitteilung) = a. 1334 plagare in den Rechnungsbüchern der Gemeinde Arbe, Mon. hist. slav. mer. V, 232, < * plagarium, von plaga Fläche REW 6562 a.

26. sul Hutweidensteuer (= solagium < *solaticum bei Du Cange VII, 5 10), mit den Ableitungen sulevina, sulisce, sulni ad., zahlreiche Belege s. bei Lopašic, Hrv. Urbari, sowie auch in Koluniceo Zbornik XXVI. < solium 4 Du Cange und REW 8079.

27. škrtt adj. knickerig, geizig, davon vb. skrtdriti, šküt(av)ac sm. Geizhals, entspricht formell dem rum. scurt, in der Bedeutung aber deckt es sich mit rum. crufa banz sparen, crufator sparsam REW 2419 sowie auch mit alb. kurtseh bin sparsam Meyer, Alb. Wbch. 216.

Agram.

Peter Skok. 